\title{
Minimally Invasive Esophagectomy with Cervical Anastomosis
}

\author{
Moshim Kukar, MD and Steven N. Hochwald, MD \\ Department of Surgical Oncology, Roswell Park Cancer Institute, Buffalo, NY
}

\begin{abstract}
This video demonstrates our technique for a minimally invasive esophagectomy with side-to-side stapled cervical esophagogastric anastomosis. This technique is routinely utilized in most patients undergoing esophagectomy for esophageal or gastroesophageal junction malignancy, excluding type III gastroesophageal junction tumors. Absolute contraindications include significant tumor involvement of the fundus which may necessitate an intrathoracic anastomosis. Relative contraindications include poor pulmonary function or prior extensive surgical history that may either preclude surgery altogether or prevent the ability of the conduit from reaching the cervical region, or could preclude utilization of a minimally invasive approach. We have not found large
\end{abstract}

body habitus to be an absolute contraindication for this approach. The technique involves thoracoscopic mobilization of the esophagus, laparoscopic dissection of the stomach and creation of gastric conduit, and creation of a $6 \mathrm{~cm}$ side-to-side stapled cervical esophagogastric anastomosis. The pylorus is treated with a botox injection; routine pyloroplasty is not performed. In our experience, this technique is safe, oncologically appropriate, and provides excellent functional results.

DISCLOSURES Dr Kukar has no relevant financial disclosures. Dr Hochwald is a consultant for Ethicon Endosurgery.

This work was presented in part at the 'Top-Rated Video Session' at the 67th Society of Surgical Oncology Annual Cancer Symposium, Phoenix, AZ, USA, 13-15 March 2014.

Electronic supplementary material The online version of this article (doi:10.1245/s10434-015-4662-5) contains supplementary material, which is available to authorized users.

(C) Society of Surgical Oncology 2015

First Received: 7 April 2015;

Published Online: 17 June 2015

M. Kukar, MD

e-mail: moshim.kukar@roswellpark.org 